\title{
Protocol
}

\section{Effect of active warming during general anaesthesia on post-operative body temperature, shivering, thermal comfort, pain, nausea and vomiting in adult patients: a randomized clinical trials protocol}

\author{
Özlem Şahin Akboğa ${ }^{1}$, Aysel Gürkan ${ }^{2}$
}

\author{
${ }^{1}$ Institute of Health Sciences, Department of Nursing, Marmara University, Turkey \\ ${ }^{2}$ Faculty of Health Sciences, Department of Nursing, Marmara University, Turkey
}

Received: 18 August 2021

Accepted: 06 October 2021

\author{
*Correspondence: \\ Özlem Şahin Akboğa, \\ E-mail: ozlemsahin.os17@gmail.com
}

Copyright: $\odot$ the author(s), publisher and licensee Medip Academy. This is an open-access article distributed under the terms of the Creative Commons Attribution Non-Commercial License, which permits unrestricted non-commercial use, distribution, and reproduction in any medium, provided the original work is properly cited.

\begin{abstract}
Background: This study aims to investigate the effects of individual and combined use of intraoperative forced-air warming and warmed intravenous and irrigation fluids on post-operative body temperature, shivering, thermal comfort, pain, nausea and vomiting in adult patients receiving general anaesthesia.

Methods: This is a randomised controlled clinical trial. A $2 \times 2$ factorial design will used in the experiment. Eligible patients will be randomly assigned to one of four groups. We attempt to report this randomized controlled trial to comply with the spirit. The study population will consist of 120 adult patients receiving general anaesthesia at university hospitals in Turkey. After the induction of anaesthesia, the first group $(n=30)$ will be warmed with only forced-air warming device, the second group $(n=30)$ will receive only warmed intravenous and irrigation fluids, forced-air warming as well as warmed intravenous (IV) and irrigation fluids will be applied to the third group $(\mathrm{n}=30)$, the fourth group $(\mathrm{n}=30)$ will consist of the control group without any intervention. Blood and blood products to be applied intraoperatively to all groups will be heated to $37^{\circ} \mathrm{C}$.

Conclusions: The primary outcome measures are post-operative body temperature, shivering, thermal comfort, pain, nausea and vomiting. The primary results will be subjected to a one-way analysis of variance for covariant such as amount of bleeding and volume of intraoperative fluids.

Trial registration: The trial has been registered in clinical trials: https://clinicaltrials.gov/. Registration number: NCT04907617).
\end{abstract}

Keywords: General anesthesia, Hypothermia, Pain, Post-operative nausea and vomiting

\section{INTRODUCTION}

Inadvertent perioperative hypothermia, defined as having a central body temperature $<36^{\circ} \mathrm{C}$, is a common and treatable adverse clinical outcome of surgery. ${ }^{1,2}$ Its incidence varies between $26 \%$ and $90 \%$, and it affects approximately $70 \%$ of the surgical patients. ${ }^{2,3}$ General anaesthesia can disrupt the thermoregulation mechanisms by blocking the communication between the central nervous system and peripheral tissues. Although changes in body temperature are usually stabilised with a standard deviation of $0.2^{\circ} \mathrm{C}$, this change can reach $6^{\circ} \mathrm{C}$ in some patients under general anaesthesia. ${ }^{4}$ This problem could be attributed to the decrease in metabolic heat generation due to anaesthetics; patients being naked and immobile in a cold operating room environment, inhaling cold gases, heat losses from body cavities, transfusion of large amounts of blood and/or blood products, heat loss caused by the use of IV and irrigation fluids and impaired thermoregulation that results in the redistribution of heat from the centre to the periphery of the body. ${ }^{5}$

Disorders in thermoregulation could lead to serious complications such as haemostatic disorders, heart 
dysfunction, liver dysfunction, coagulation disorders, renal dysfunction, immune dysfunctions or delayed wound healing. ${ }^{6}$ In addition, the prolonged hospital and intensive care unit, stay results in increased costs and decreased patient satisfaction. ${ }^{7-9}$ Therefore, prevention of inadvertent perioperative hypothermia is a high priority patient safety issue. ${ }^{10}$

Adult patients who undergo surgery under general anaesthesia often experience inadvertent perioperative hypothermia., 5,11 In evidence-based guidelines, it is recommend to use a range of active and passive warming methods with close monitoring of the patient's body temperature for maintaining peri-operative normothermia. ${ }^{1,12,13}$ Among the active warming methods, hot forced-air warming is the most effective method. Another method recommended for the prevention of intraoperative hypothermia is the administration of warmed IV and irrigation fluids. ${ }^{9,14}$ In a Cochrane review, it was shown that in adult patients who underwent abdominal surgery under general anaesthesia, forced-air warming provided before or during the operation or in both periods had a beneficial effect in terms of reduced surgical site infections and complications, cardiovascular complications and shivering. Besides, this method increased the thermal comfort of patients compared to those without any active warming system. ${ }^{1}$ In another Cochrane review, intraoperative administration of heated IV and irrigation fluids had a beneficial effect on postoperative body temperature and shivering. However, it was concluded that the clinical significance of this benefit was unclear because of the use of the method in addition to other active warming methods. ${ }^{15}$ Both interventions are clearly effective with varying levels of evidence in maintaining intraoperative normothermia and improving post-operative complications. These interventions have no reported side effects. ${ }^{1,15}$ However, well-designed studies comparing the effectiveness of the methods are needed to clearly determine the clinical benefit.

In studies, the effect of active warming on body temperature, shivering and thermal comfort have been frequently examined, while little attention has been paid to the effects of active warming on post-operative pain, nausea and vomiting. ${ }^{13,16-19}$ However, preventing these problems commonly experienced in the early postoperative period is very important in accelerating the surgical recovery.

The first physiological response of inadvertent perioperative hypothermia is shivering. Shivering develops in $5 \%-65 \%$ of patients operated under general anesthesia and is seen 3.3 times more in hypothermic patients after surgery than in normothermic patients. ${ }^{13,20}$ Shivering elevates the risk of hypoxemia by increasing oxygen consumption ${ }^{21}$ and triggers post-operative pain, nausea and vomiting. ${ }^{21,22}$ With shivering, the severity of these complications increases owing to the effect of various drugs (anaesthetics, opioids, antibiotics, etc.). ${ }^{23}$ In addition, shivering disrupts the patient's thermal comfort, which leads to deterioration of general comfort and decrease in the satisfaction with care. ${ }^{5,9}$

The relationship between shivering and pain was first mentioned by Sessler et al. It is thought that pain experienced along the surgical incision line may cause post-operative shivering. While shivering is facilitated by pain, shivering also exacerbates pain. ${ }^{21,22}$ Studies showing higher pain severity in hypothermic patients compared to normothermic patients support this view. ${ }^{19}$

Another unpleasant complication in patients undergoing surgery under general anaesthesia is post-operative nausea and vomiting. ${ }^{24}$ Although the relationship between post-operative nausea and vomiting and thermoregulation mechanisms is little known, the incidence of nausea is $50 \%$ and the incidence of vomiting is $>20 \% .^{25,26}$ These rates are $>30 \%$ in hypothermic patients when compared to normothermic patients. ${ }^{13}$

Post-operative shivering, thermal comfort, pain, nausea and vomiting are not life-threatening complications, but they must be prevented and treated as they affect the general comfort and care satisfaction of the patient. ${ }^{27}$ Perioperative nurses have important roles in the prevention and treatment of these complications. ${ }^{12}$

In this context, this trial will examine the effects of warming the patient intraoperatively with a forced-air warming device and the administration of warmed IV and irrigation fluids on patient outcomes that concern perioperative nurses, namely post-operative body temperature, shivering, thermal comfort, pain, nausea and vomiting.

\section{Hypotheses}

H1-Only forced-air warming device will better protect the post-operative body temperature and will be increased thermal comfort and will be decreased the post-operative shivering, pain level and the presence of post-operative nausea and vomiting of other groups (group 2, 3 and 4) with statistically significant differences.

H2-Only warmed intravenous and irrigation fluids will better protect the post-operative body temperature and will be increased thermal comfort and will be decreased the post-operative shivering, pain level and the presence of post-operative nausea and vomiting of other groups (group 3 and 4) with the statistically significant differences.

H3-Forced-air warming as well as warmed intravenous (IV) and irrigation fluids will better protect the postoperative body temperature and will be increased thermal comfort and will be decreased the post-operative shivering, pain level and the presence of post-operative nausea and vomiting of other group (group 4) with statistically significant differences. 
Aim

The main purpose of this study is to determine the effect of individual and combined administration of intraoperative forced-air warming and heated IV and irrigation fluids on post-operative body temperature, shivering, thermal comfort, pain, nausea and vomiting in adult patients receiving general anaesthesia.

The secondary objectives are to determine the effects of these interventions on the patient's post-operative analgesic and anti-emetic requirement and the presence of complications such as bleeding, fever and atelectasis.

\section{METHODS}

\section{Determination of the sample size}

For this trial, the sample size will be calculated by power analysis using the $G^{*}$ power (v3.1.7) programme. Since the power of the study will be expressed as $1-\beta(\beta=$ Type II error probability) and the studies should have $80 \%$ power, the sample number will be determined in the trial according to the Cohen's effect size coefficient. Accordingly, assuming that the evaluations to be made between two independent groups will have a large effect size $(d=0.8)$, there should be at least 26 people $(n=104)$ in each group. Thirty people will be included $(n=120)$ in each group.

\section{Ethical considerations}

This study will be conducted in accordance with the principles of the declaration of Helsinki (DTB general assembly, Fortaleza, Brazil, October 2013) and the medical research involving human subjects act, and written informed consent will be obtained from all participants throughout the research. This research was approved by the Clinical Research Ethics Committee of Marmara University Faculty of Medicine (Protocol number: 09.2020/770). Institutional permission was obtained from Yozgat Bozok University Research and Application Centre in which the research will be conducted (Date:14.05.2020, number:16142545-903.99E.10438).

\section{Population and randomization}

This study protocol describes the design of a singlecentre, single-blind and randomized controlled trial. This trial will be conducted in the operating room and surgical wards of a university hospital in Yozgat, Turkey. The study is registered at clinicaltrials.gov in June 2021 (NCT04907617). The study was planned four groups. At the beginning of the study, the assignment sequence of patients who meet the inclusion criteria will be created with a random number generator (https://www.randomizer.org). The participants in the study will be allocated to one of four groups. In the trial, the participants will be assigned to the interventions and registered by the first author of this study. In this randomised controlled trial, a $2 \times 2$ factor design will be used in which each group will be randomly assigned an equal number of participants (Table 1). The design will allow an examination of the effects of each intervention on post-operative body temperature, shivering, thermal comfort, pain, nausea and vomiting as well as the effects of using these interventions individually and in combination.

Table 1: Interventions allocated to each study group.

\begin{tabular}{|lll|}
\hline Variables & $\begin{array}{l}\text { Warmed IV and } \\
\text { irrigation } \\
\text { solutions }\end{array}$ & $\begin{array}{l}\text { No warmed IV } \\
\text { and irrigation } \\
\text { solutions }\end{array}$ \\
\hline $\begin{array}{l}\text { Forced air } \\
\text { warming }\end{array}$ & Group 3 & Group 1 \\
\hline $\begin{array}{l}\text { No forced } \\
\text { air warming }\end{array}$ & Group 2 & Group 4 \\
\hline
\end{tabular}

Inclusion and exclusion criteria were established in accordance with the literature. . $^{16,29,30}$

\section{Inclusion criteria}

The patients with 18 years of age or older, received general anesthesia, surgery that lasted $>1$ hour, the class of American society of anaesthesiologists I-III (Table 2) and being able to communicate in Turkish will be included in the study.

\section{Exclusion criteria}

Patients having a pre-operative body temperature of $\geq 37.5^{\circ} \mathrm{C}$ or $<36^{\circ} \mathrm{C}$, having undergone a surgical intervention that prevents measurement of body temperature by the tympanic route, having received intraoperative therapeutic hypothermia, having systemic infection, mental retardation, deterioration in temperature regulation such as isolated head trauma or brain injury, having received a drug therapy that would affect thermoregulation, having received heated IV fluid therapy up to one hour before anaesthesia induction will not be included in the study and patients who become unstable or exitus during and after the operation will be excluded from the study.

Table 2: American society of anesthesiologists grading.

\begin{tabular}{|c|c|}
\hline ASA & Description \\
\hline I & Healthy individual with no systemic disease \\
\hline II & Mild systemic disease not limiting activity \\
\hline III & $\begin{array}{l}\text { Severe systemic disease that limits activity but } \\
\text { is not incapacitating }\end{array}$ \\
\hline IV & $\begin{array}{l}\text { Severe systemic disease that limits activity but } \\
\text { is not incapacitating. }\end{array}$ \\
\hline $\mathbf{V}$ & $\begin{array}{l}\text { Incapacitating systemic disease which is } \\
\text { constantly life-threatening }\end{array}$ \\
\hline
\end{tabular}




\section{Blinding}

The participants will be blinded to the trial hypothesis and design to avoid being affected by the warming intervention. $^{31}$ This aspect of the study has a "singleblind" design.

\section{Interventions}

After the induction of anaesthesia, forced-air warming as well as warmed IV and irrigation fluids will be applied to the first group, the second group will be heated with only forced-air warming device, the third group will receive only warmed IV and irrigation fluids and the fourth group will be the control group without any intervention.

After the induction of anaesthesia, for patients assigned to groups 1 and 2, the cover of the forced-air warming device (Bair HuggerTM Warming Unit, Model 505, Augustine Medical Inc., Eden Prairie, MN, USA) will be covered over the patient, with the surgical site exposed, and the temperature adjustment of the device will be set to the maximum $\left(43^{\circ} \mathrm{C}\right)$. Then, the temperature will be adjusted to maintain a patient temperature of at least $36.5^{\circ} \mathrm{C} .{ }^{14}$ When the patient's body temperature reaches $37^{\circ} \mathrm{C}$, the device will be turned off and it will be restarted at a temperature measurement of $<36.5^{\circ} \mathrm{C}$. ${ }^{5}$

In patients assigned to groups 2 and 3, intraoperative IV (Automer blood/fluid warming system, Acemedikal, South Korea) and irrigation fluids (Enthermic Warming Cabinet $1002 \mathrm{~W}$, Poland) will be given by heating to $37^{\circ} \mathrm{C}$ in a thermostatically controlled heating cabinet. ${ }^{32}$

The patients assigned to group 4 will only receive 'routine care'. They will not be given intraoperative forced-air warming or heated IV and irrigation fluids.

In all the groups, blood and/or blood products to be administered in case of intraoperative need, will be heated to $37^{\circ} \mathrm{C}$ with a heating system (Automer blood/fluid warming system, Acemedikal, South Korea), ${ }^{9,11}$ and the intraoperative body temperature will be monitored at 30-minute intervals. ${ }^{5,9,11}$

\section{Outcomes}

The data collection instrument, the individual and clinical features form and patient follow-up form was designed specifically for this study in accordance with the literature. $^{5,11,32}$ This instrument used to record demographic information (age, gender, diagnosis, type of surgery, ASA class, presence of chronic disease, length of stay in hospital etc.) and outcome variables. After obtaining consent from the participants, sociodemographic and clinical data will be gathered. Data on outcomes will be collected by a first researcher. The participants will be blinded as to the group assignment.

\section{Primary outcome measure}

The primary outcomes of this trial are post-operative body temperature, shivering, thermal comfort, pain, nausea and vomiting.

\section{Body temperature}

In the trial, the body temperature of the participants will be measured with a tympanic thermometer (Braun ThermoScan ${ }^{\circledR}$ 3, IRT 3030, Mexico, USA). This thermometer will be calibrated at regular intervals by the biomedical department of the hospital where the study is conducted, according to the manufacturer's instructions.

\section{Thermal comfort}

The thermal comfort of the patients will be measured with a 100-point thermal comfort scale based on selfreporting. The patients will be asked to score how comfortable they are with their body temperature $[0=$ extremities too cold; $50=$ no discomfort (shivering or sweating); $100=$ extremities too hot] on a scale of $0-100$ points. ${ }^{33}$ This numerical rating scale has been used effectively in previous studies on thermal comfort. ${ }^{18,33}$

\section{Shivering}

Shivering will be assessed using a 4-point bedside shivering assessment scale developed by Badjatia et al $[0=$ no shivering; $1=$ mild (shivering localised in the neck/thorax, only visible as an artefact on EEG or can be noted on palpation); $2=$ moderate (intermittent shivering in the upper extremities \pm thorax); $3=$ severe (diffuse shivering or continuous shivering in the upper/lower extremities)]. ${ }^{34}$ The scale has been used effectively in previous studies on hypothermia. ${ }^{18}$

\section{Post-operative pain}

The post-operative pain of the patients will be assessed with the numerical rating scale (NRS) based on selfreporting. The obtained NRS score will be considered as $0=$ no pain, $1-3=$ mild pain, $4-6=$ moderate pain, 7$10=$ severe pain. The confidence interval of NRS, which is one of the scales recommended for the assessment of acute pain in adults, is determined as $100 \%$. $^{35,36}$

\section{Post-operative nausea and vomiting}

Based on a previous study, the presence of post-operative nausea and vomiting of the patients in the trial will be considered as follows: $0=$ no nausea; $1=$ nausea without vomiting; $2=$ nausea with vomiting. ${ }^{18}$

\section{Secondary outcome measure}

The secondary outcomes are ambient temperature in preoperative waiting unit, operating room, post-anaesthesia care unit (PACU) and surgical clinics, patient's blood pressure, heart rate and respiratory rate, duration of 
operation of the intraoperative forced-air warming device, volume of IV and irrigation fluids delivered, the amount of bleeding present and the amount of blood and blood products applied by heating when required, postoperative analgesic and anti-emetic drugs the status of applying blood and blood products, and the presence of complications such as fever, bleeding and atelectasis. $^{17,19,29,30}$

\section{Discharge from the PACU}

Eligibility for discharge from PACU will be evaluated using the Modified Aldrete Score (MAS), which is a standardised post-anaesthesia discharge scoring tool (Table 3). ${ }^{37,38}$

Table 3: The Modified Aldrete Score (MAS) system.

\begin{tabular}{|c|c|c|}
\hline $\begin{array}{l}\text { Assessment } \\
\text { items }\end{array}$ & Condition & Grade \\
\hline \multirow{3}{*}{$\begin{array}{l}\text { Activity, able } \\
\text { to move, } \\
\text { voluntarily or } \\
\text { on command }\end{array}$} & 4 extremities & 2 \\
\hline & 2 extremities & 1 \\
\hline & No & 0 \\
\hline \multirow{3}{*}{ Breathing } & $\begin{array}{l}\text { Able to breathe deeply and } \\
\text { cough freely }\end{array}$ & 2 \\
\hline & $\begin{array}{l}\text { Dyspnea, shallow or limited } \\
\text { breathing }\end{array}$ & 1 \\
\hline & Apnea & 0 \\
\hline \multirow{3}{*}{$\begin{array}{l}\text { Circulation } \\
\text { (BP) }\end{array}$} & $\pm 20 \%$ of pre-anesthesia level & 2 \\
\hline & $\begin{array}{l} \pm 20-49 \% \text { of pre-anesthesia } \\
\text { level }\end{array}$ & 1 \\
\hline & \pm 50 of pre-anesthesia level & 0 \\
\hline \multirow{3}{*}{ Consciousness } & Fully awake & 2 \\
\hline & Arousable on calling & 1 \\
\hline & Unresponsive & 0 \\
\hline \multirow{3}{*}{$\mathrm{SpO}_{2}$} & $\begin{array}{l}\text { Maintains } \mathrm{SpO}_{2}>92 \% \text { in } \\
\text { ambient air }\end{array}$ & 2 \\
\hline & Maintain $\mathrm{SpO}_{2}>90 \%$ with $\mathrm{O}_{2}$ & 1 \\
\hline & Maintain $\mathrm{SpO}_{2}<90 \%$ with $\mathrm{O}_{2}$ & 0 \\
\hline Total & & 15 \\
\hline
\end{tabular}

\section{Data collection procedure}

All patients consecutively admitted to the surgical clinic will be screened in terms of the inclusion criteria; patients who meet the inclusion criteria will be invited to the study and will be included in the study after obtaining informed written consent. In this process, the demographic and clinical information of the patient will be collected. The baseline measurement of the patients' body temperature will be made in the pre-operative waiting unit (T0). Follow-up measurements will be collected at 13 time points, as follows: just before anaesthesia induction (T1) and every 30 min during the surgery (T2 1, 2 and 3...), at admission to PACU (T3), 30 min after admission to PACU (T4), before discharge from PACU (T5), at admission to the surgical clinic (T6) and 30 min (T7) and 1 (T8), 2 (T9), 3 (T10), 4 (T11), 12
(T12) and $24 \mathrm{~h}$ after admission to the clinic (T13). The baseline measurement of the patient's thermal comfort (T0) will be performed in the pre-operative waiting unit, while the follow-up measurements will be carried out along with the body temperature measurements at PACU and surgical clinic (T3-T13). Post-operative shivering, pain, nausea and vomiting measurements of the patient will be made simultaneously with the body temperature and thermal comfort measurements at PACU and surgical clinic (T3-T13). Details and timing of data collection in the study is shown in Figure 1.

In this process, all secondary parameters will be evaluated as follows: the ambient temperature in the preoperative waiting unit, operating room, PACU and surgical clinics and the patient's blood pressure, heart rate and respiratory rate, in these environments; the operating time of the intraoperative forced-air warming device; the volume of IV and irrigation fluids, the amount of bleeding present and the amount of blood and/or blood products warmed if required; MAS at discharge from PACU; analgesic and anti-emetic drugs administered up to 48 hours post-operatively; the status of administering blood and blood products; and presence of complications such as fever, bleeding and atelectasis will be recorded. ${ }^{1,7,17,19,30,32}$

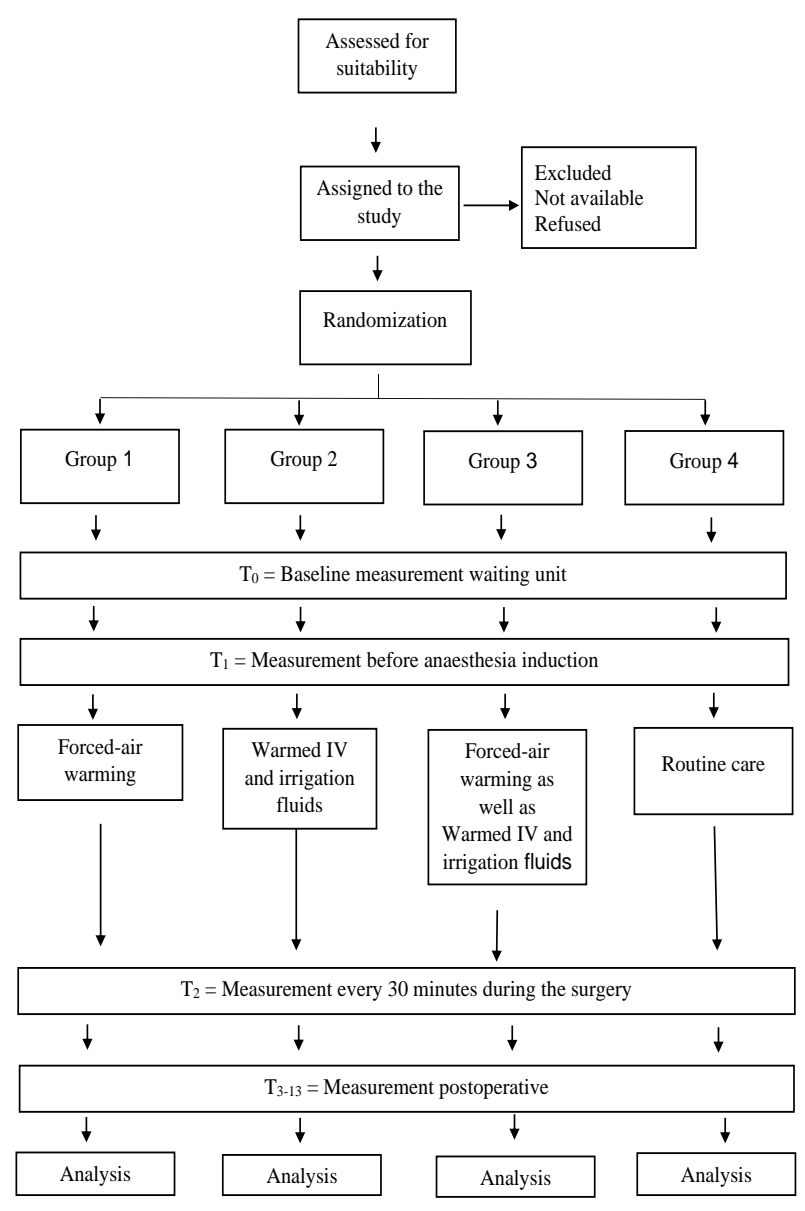

Figure 1: Data collection procedure. 


\section{Data analysis}

The data will be analysed using statistical software (IBM® SPSS $®$ Statistics version 21$)$. While evaluating the study data, descriptive statistical methods (ratio, mean, standard deviation and median) as well as ShapiroWilk test and box-plot graphs will be used to ensure the compliance of the variables with the normal distribution. Paired two-sample t-test will be used to compare the normally distributed data according to pairwise time within the group, and Wilcoxon test will be used to compare the data that is not normally distributed. McNemar test will be used to compare two-state categorical data according to in-group binary time, and McNemar-Browker test will be used to compare categorical variables with three or more states. The Friedman test will be used to compare data that is not normally distributed over three or more times. Pearson correlation coefficient will be used to analyze the relationship between normally distributed data, and Spearman's rho correlation coefficient will be used to analyze the relationship between non-normally distributed data.Paired two-sample t-test will be used to compare the normally distributed data according to pairwise time within the group, and Wilcoxon test will be used to compare the data that is not normally distributed. McNemar test will be used to compare two-state categorical data according to in-group binary time, and McNemar-Browker test will be used to compare categorical variables with three or more states. The Friedman test will be used to compare data that is not normally distributed over three or more times. Pearson correlation coefficient will be used to analyze the relationship between normally distributed data, and Spearman's rho correlation coefficient will be used to analyze the relationship between non-normally distributed data. Chi-square test and Fisher's exact test will be used to compare qualitative data. Results will be evaluated at $95 \%$ confidence interval and a significance level of $\mathrm{p}<0.05$.

\section{Primary study parameter}

The basic analysis is the comparison of post-operative body temperature, shivering, thermal comfort, pain, nausea and vomiting between the groups. Generalised linear models will be used in repeated measurements.

\section{Secondary study parameter}

Shapiro-Wilk test and box-plot graphs will be used in analysing the conformity of the variables to normal distribution, such as duration of operation, volume of intraoperative heated blood/fluids measure the blood pressure, heart rate, respiratory rate, saturation and amount of blood loss, MAS score, amount of analgesic and anti-emetic applied up to 48 hours post-operatively, the amount of blood and blood products administered, the presence of complications. For normally distributed variables, one-way ANOVA test, and for variables that do not show normal distribution, Kruskal-Wallis test will be used.

\section{DISCUSSION}

Inadvertent perioperative hypothermia, a common clinical consequence of general anaesthesia, has serious adverse consequences ranging from the patient's thermal discomfort to increased morbidity and possible mortality. ${ }^{9,14}$ Despite the current technological and scientific advances in its prevention and management, inadvertent perioperative hypothermia is often overlooked in practice and remains to be a problem during the perioperative period..$^{9,11}$ National and international evidence-based guidelines recommend the use of intraoperative forced-air warming and warmed IV and irrigation fluids in adult patients undergoing surgery under general anaesthesia. ${ }^{5,9,11}$ While these methods have varying levels of evidence in achieving normothermia and alleviating post-operative complications, quality studies comparing the effectiveness of the methods are needed to clearly determine the clinical benefit. ${ }^{1,15}$ In addition, although the effects of these interventions on post-operative body temperature, shivering and thermal comfort have been studied frequently, their effects on pain, nausea and vomiting have been investigated only in a limited number of studies. ${ }^{18,21,25}$ Peri-operative nurses have important responsibilities in the prevention and treatment of these post-operative complications. In this context, perioperative nurses are critical in determining the best practice in preventing hypothermia and achieving normothermia. ${ }^{39}$

\section{CONCLUSIONS}

This study is the first trial to meticulously evaluate the effects of warming the patient with intraoperative forcedair warming and the administration of heated IV and irrigation fluids on the outcomes with which the perioperative nurses are concerned in accelerating recovery. These outcomes include body temperature, shivering, thermal comfort, pain, nausea and vomiting. In addition, the research design will provide valuable evidence for perioperative clinical practice and provide a one-to-one comparison of the effects of using these interventions alone and in combination.

\section{ACKNOWLEDGEMENTS}

Author would like to thanks Prof. Dr. Ahmet Sebe and Prof. Dr. Ferit Çiçekçioğlu, who assisted us in the supply of devices, operating room, surgical clinic nurses and doctors as well as for their support in providing information and guidance.

\section{Funding: No funding sources}

Conflict of interest: None declared

Ethical approval: The study was approved by the Institutional Ethics Committee by Marmara University Faculty of Medicine (Protocol number: 09.2020/770). 
Institutional permission was obtained from Yozgat Bozok University Research and application Centre (Date:14.05.2020, number:16142545-903.99-E.10438).

\section{REFERENCES}

1. Madrid E, Urrútia G, Roqué Figuls M, PardoHernandez H, Campos JM, Paniagua P et al. Active body surface warming systems for preventing complications caused by inadvertent perioperative hypothermia in adults. Cochrane Database Systematic Rev. 2016:4:CD009016.

2. Torossian A, Bräuer A, Höcker J, Bein B, Wulf H, Horn EP. Clinical practice guideline: Preventing inadvertent perioperative hypothermia. Dtsch Arztebl Int. 2015;112:166-72.

3. Giuliano KK, Hendricks J. Inadvertent perioperative hypothermia: Current nursing knowledge. AORN J. 2017;105(5):453-63.

4. Bindu B, Bindra A, Rath G. Temperature management under general anesthesia: Compulsion or option. J Anaesthesiol Clin Pharmacol. 2017;33:306-16.

5. Anestezi T, Reanimasyon Derneği V, TARD. Anestezi uygulama klavuzları: İstenmeyen perioperatif hipoterminin önlenmesi rehberi. Turk $\mathbf{J}$ Anaesth Reanim. 2013;41:188-90.

6. Iden T, Höcker J. Prevention of Perioperative Hypothermia-Guidelines for Daily Clinical Practice. Anasthesiol Intensivmed Notfallmed Schmerzther. 2017;52:554-62.

7. Jeyadoss J, Thiruvenkatarajan V, Watts RW, Sullivan T, Van Wijk RM. Intraoperative hypothermia is associated with an increased intensive care unit length-of-stay in patients undergoing elective open abdominal aortic aneurysm surgery: A retrospective cohort study. Anaesth Intensive Care. 2013;41(6):759-64.

8. Lista F, Doherty CD, Backstein RM, Ahmad J. The impact of perioperative warming in an outpatient aesthetic surgery setting. Aesthet Surg J. 2012;32(5):613-20.

9. Hooper VD, Chard R, Clifford T, Fetzer S, Fossum S, Godden B et al. ASPAN's evidence-based clinical practice guideline for the promotion of perioperative normothermia: Second edition. J Perianesth Nurs. 2010;25(6):346-65.

10. Steelman VM, Graling PR, Perkhounkova Y. Priority patient safety issues 1dentified by perioperative nurses. AORN J. 2013;97(4):402-18.

11. Association of periOperative Registered Nurses, (AORN). 2019. Available at: https://www.aorn.org/guidelines/about-aornguidelines/evidence-tables. Accessed on 13 July 2021.

12. Su SF, Nieh HC. Efficacy of forced-air warming for preventing perioperative hypothermia and related complications in patients undergoing laparoscopic surgery: A randomized controlled trial. Int $\mathrm{J}$ Nurs Pract. 2018;24(5):e12660.
13. Aydın H, Şimşek T, Demiraran Y. Effects of 1nadvertent perioperative hypothermia on metabolic and inflammatory mediators. Turk J Anaesthesiol Reanim. 2019;47(6):448-55.

14. Riley C, Andrzejowski J. Inadvertent perioperative hypothermia. BJA Education. 2018;18(8): 227e233.

15. Campbell G, Alderson P, Smith AF, Wortting S. Warming of intravenous and irrigation fluids $\mathrm{fr}$ preventing inadvertent perioperative hypothermia. Cochrane Database Syst Rev. 2015;13(4):CD009891.

16. Choi JW, Kim DK, Lee SW, Park JB, Lee GH. Efficacy of intravenous fluid warming during goaldirected fluid therapy in patients undergoing laparoscopic colorectal surgery: A randomized controlled trial. J Int Med Res. 2016;44(3):605-12.

17. Kim YS, Lee JY, Yang SC, Song JH, Koh HS, Park WK. Comparative study of the influence of roomtemperature and warmed fluid irrigation on body temperature in arthroscopic shoulder surgery. Arthroscopy: J Arthroscopic Related Sur. 2009;25(1):24-9.

18. Ayhan S, Ballı SS, Firat AC, Kayhan Z. Spinal anestezi ile sezeyan ameliyatı uygulanan gebelerde hipotermi önlenebilir mi? J Anesthesia. 2018;26(4):238-44.

19. Benson EE, McMillan DE, Ong B. The effects of active warming on patient temperature and pain after total knee arthroplasty. Am J Nurs. 2012;112 (5):2633.

20. Buggy DJ, Crossley AW. Thermoregulation, mild perioperative hypothermia and post-anaesthetic shivering. Br Journal Anaesth. 2000;84:615-28.

21. Lopez MB. Postanaesthetic shivering- from pathophysiology to prevention. Rom J Anaesthesia Intensive Care. 2018;25(1):73-81.

22. Sessler DI, Rubinstein EH, Moayeri A. Physiologic responses to mild perianesthetic hypothermia in humans. Anesthesiology. 1991;75:594-610.

23. Kim D. Postoperative Hypothermia. Acute Crit Care. 2019;34(1):79-80.

24. Mohammadi SS, Jabbarzadeh S, Movafegh A. Efficacy of granisetron on prevention of shivering, nausea and vomiting during cesarean delivery under spinal anesthesia: A randomized double-blinded clinical trial. J Obstetr Anaesthes Cri Care. 2015;5(1):22-6.

25. Nalivaiko E. Thermoregulation and nausea. Handb Clin Neurol. 2018;156:445-56.

26. De Boer HD, Detriche O, Forget P. Opioid-related side effects: Postoperative ileus, urinary retention, nausea and vomiting, and shivering. Best Pract Res Clin Anaesthesiol. 2017;31(4):499-504.

27. Sessler DI. Perioperative Thermoregulation and heat balance. Lancet. 2016;25,387 (10038):2655-64.

28. Su SF, Nieh HC. Efficacy of forced-air warming for preventing perioperative hypothermia and related complications in patients undergoing laparoscopic surgery: A randomized controlled trial. Int $\mathrm{J}$ Nurs Pract. 2018;24(5):e12660. 
29. Fanelli A, Danelli G, Ghisi D, Ortu A, Moschini E, Fanelli $\mathrm{G}$. The efficacy of a resistive heating underpatient blanket versus a forced-air warming system: A randomized controlled trial. Anesthesia Analgesia. 2009;108(1):199-201.

30. Duff J, Di Staso R, Cobbe KA, Draper N, Tan S, Halliday $\mathrm{E}$ et al. Preventing hypothermia in elective arthroscopic shoulder surgery patients: A protocol for a randomised controlled trial. BMC surgery. 2012;12(1):1-6.

31. Boutron I, Guittet L, Estellat C, Moher D, Hróbjartsson A, Ravaud P. Reporting methods of blinding in randomized trials assessing nonpharmacological treatments. PLoS Med. 2007;4(2):e61.

32. National Institute for Health and Clinical Excellence. NICE. Inadvertent perioperative hypothermia overview, 2021. Available at: https://pathways.nice. org.uk/pathways/inadvertent-perioperativehypothermia\#path=view\%3A/ pathways/inadvertentperioperative-hypothermia/inadvertent-perioperativehypothermia-overview.xml\&content=view-node $\% 3$ Anodes-intraoperative-phase. Accessed on 13 July 2021.

33. Cobb B, Cho Y, Hilton G, Ting V, Carvalho B. Active warming utilizing combined IV fluid and forced-air warming decreases hypothermia and improves maternal comfort during cesarean delivery: A randomized control trial. Anesth Analg. 2016;122:1490-7.
34. Badjatia N, Strongilis E, Gordon E, Prescutti M, Fernandez L, Fernandez A, et al. Metabolic impact of shivering during therapeutic temperature modulation: The Bedside Shivering Assessment Scale. Stroke. 2008;39(12):3242-7.

35. Chou R, Gordon DB, de Leon-Casasola OA, Rosenberg JM, Bickler S, Brennan $\mathrm{T}$ et al. Guidelines on the management of postoperative pain. J Pain. 2016;17(2):131-57.

36. Odejobi YO, Maneewat K, Chittithavorn V. Nurseled post-thoracic surgery pain management programme: İts outcomes in a Nigerian Hospital. Int Nurs Rev. 2019;66(3);434-41.

37. Aldrete JA, Kroulik D. A postanesthetic recovery score. Anesth Analg. 1970;49(6): 924-34.

38. Aldrete JA. The post-anesthesia recovery score revisited. J Clin Anesth. 1995;7:89-91.

39. Bender M, Self B, Schroeder E, Giap B. Comparing new-technology passive warming versus traditional passive warming methods for optimizing perioperative body core temperature. AORN J. 2015;102(2):183.e1-8.

Cite this article as: Akboğa OS, Gürkan A. Effect of active warming during general anaesthesia on postoperative body temperature, shivering, thermal comfort, pain, nausea and vomiting in adult patients: a randomized clinical trials protocol. Int J Clin Trials 2021;8(4):315-22. 